\title{
Changes in cerebral tissue perfusion during the first 48 hours of ischaemic stroke: relation to clinical outcome
}

\author{
A E Baird, M C Austin, W J McKay, G A Donnan
}

\begin{abstract}
Background-One major therapeutic strategy to minimise the extent of infarction after ischaemic stroke is to improve early reperfusion using thrombolytic agents. However, reperfusion may be hazardous and the period during which reperfusion may have a beneficial effect on tissue and clinical outcome is not known.

Methods-Fifty three patients were studied with serial cerebral perfusion ${ }^{99 \mathrm{~m}} \mathrm{Tc}$ HMPAO SPECT) during the first 48 hours of ischaemic stroke to determine if changes in tissue perfusion during this time were prognostically significant. Single and multiple linear regression non-parametric analyses were used to include other factors during the same period which may influence outcome.

Results-In univariate analysis age, neurological score at admission, SPECT perfusion defect size in the first 24 hours, and percentage change in cerebral tissue perfusion at 24-48 hours (all $P<0.01$ ) correlated significantly with the Barthel score at three months. In multiple linear regression analysis only age $(P<0.01)$ and percentage change in cerebral tissue perfusion at $24-48$ hours $(P<0.01)$ provided independent prognostic information at three months.

Conclusions-changes in cerebral tissue perfusion during the first 48 hours of ischaemic stroke are significant outcome predictors and therapeutic efforts aimed at increasing perfusion during this period seem to be justified.
\end{abstract}

Neurology

A E Baird

G A Donnan

Department of Nuclear

Medicine, Austin

Hospital, Victoria,

Australia

M C Austin

W J McKay

University of Melbourne, Australia

A E Baird

G A Donnan

Correspondence to:

Professor G A Donnan,

Department of Neurology,

Austin Hospital, Heidelber

(Melbourne), Victoria 3084

Australia.

Received 25 April 1995 and in final revised form 25 January 1996

Accepted 22 March 1996 sion may be accompanied by clinical recovery has yet to be determined. ${ }^{2}$ In addition, salvage of the ischaemic penumbra may not appreciably reduce infarct size or improve outcome if the penumbra is confined to a narrow rim of tissue.

Reperfusion in the first 24 to 48 hours of cerebral ischaemia has been found in a limited number of SPECT and PET studies with conflicting findings. In some reperfusion was associated with a beneficial clinical outcome, ${ }^{3-5}$ in one other the association was variable,${ }^{6}$ and in another there was no clinical benefit. ${ }^{7}$ Reasons for the conflicting findings may include: (a) small patient numbers, ${ }^{5-6}(b)$ different degrees of nutritional and non-nutritional reperfusion, (c) lack of semiquantitative analysis of data, ${ }^{6}$ (d) inference that reperfusion had occurred in individual patients based on a single scan only, ${ }^{3}(e)$ different time windows studied ranging from 18 hours $^{3}$ to 48 hours, ${ }^{4-57}$ and $(f)$ failure to take into account other factors which may influence outcome such as age and neurological deficit. ${ }^{8-13}$

To clarify this important issue, we have prospectively studied 53 patients with sequential SPECT within 48 hours of acute ischaemic stroke and then used multiple linear regression non-parametric analysis to account for other factors which may influence clinical outcome. ${ }^{14}$

\section{Method}

\section{STUDY ESTABLISHMENT}

The study was conducted prospectively from April 1991 through to October 1993. Patients admitted to the Austin Hospital stroke unit ${ }^{15}$ with the sudden onset of a focal neurological event consistent with ischaemic stroke, who underwent serial ${ }^{99 m}$ Tc-HMPAO (hexamethylpropyleneamine oxime) SPECT studies during the first 48 hours of ischaemia (see below), who had abnormal perfusion images, and who did not have recurrent stroke during the follow up period, were included in the study. All patients had a non-contrast brain CT on admission and after seven days, or earlier if clinically indicated. Patients with reversible ischaemic neurological deficits (defined as lasting longer than 24 hours) were included if their perfusion study at admission was abnormal. Patients with prior stroke were included if they had made a full functional recovery and the current ischaemic locus was in a site remote from the current site. If the neurological 
deficit was first noticed on awakening, the time of onset was back dated to the time the patient was last clinically normal. Some patients were participants in the pilot and randomised phases of the Australian streptokinase trial. ${ }^{16}$

\section{CLINICAL ASSESSMENT}

The validated modified Canadian neurological score (MCNS) ${ }^{17}$ was used as the measure of neurological disability on admission. Outcome assessment was based on the Barthel index ${ }^{18}$ at three months. This weighted disability scale has been extensively validated. ${ }^{19}$ The maximum score of 100 indicates full functional independence. Deceased patients were given a score of zero. Other clinical variables recorded were age, sex, and history of hypertension or heart disease. "Hypertension" was defined as a medical history of treated hypertension. Patients who were hypertensive at the time of presentation were not classified as "hypertensive" as patients may be hypertensive in the acute phase of ischaemic stroke. ${ }^{820}$ "Heart disease" was defined as cardiac abnormalities based on information obtained from the medical history, clinical examination, and results of ECG and echocardiogram recordings. These included conditions such as atrial fibrillation, rheumatic valvar heart disease, ischaemic heart disease, and cardiomyopathy.

\section{FUNCTIONAL IMAGING}

99mTc-HMPAO SPECT (15-25 mCi, CeretecAmersham, Australasia) was performed with a rotating General Electric 400 AC Starcam camera (Milwaukee USA) during the working hours of the nuclear medicine department. Sixty four images were acquired over $360^{\circ}$ on a $128 \times 128$ matrix with a pixel size of $3.1 \mathrm{~mm}$ and acquisition time of 15-30 s/ frame. After scatter correction and attenuation correction using an attenuation coefficient of $0 \cdot 15,,^{21}$ slices one pixel thick were reconstructed on a $64 \times 64$ matrix. Image resolution measured in a resolution phantom was $12 \mathrm{~mm}$ at full width half maximum.

Repeat ${ }^{99 \mathrm{~m}} \mathrm{Tc}-\mathrm{HMPAO}$ and scanning were performed about 24 hours after the initial 99m Tc-HMPAO study to measure the extent of perfusion change. The dose, time of injection, time of scanning, head position during scanning, and count rate were matched as closely as possible for each scan. No correction was made for residual activity that may have still been present at the time of the second study.

Semiquantitative volumetric analysis of size and severity of the perfusion defect was per-

Table 1 Demographic details of the 53 patients in the study

\begin{tabular}{ll}
\hline Variable & \\
\hline Age (y) & $69 \cdot 3(1 \cdot 6)$ \\
Sex (M/F) & $23 / 30$ \\
Admission neurological score & $4 \cdot 3(0 \cdot 4)$ \\
Hypertension (n) & 33 \\
Heart disease (n) & 35 \\
Perfusion defect size in first 24 hours $\left(\mathrm{cm}^{3}\right)$ & $64 \cdot 3(7 \cdot 2)$ \\
Percentage perfusion change at $24-48$ hours $(\%)$ & $30 \cdot 6(7 \cdot 0)$ \\
\hline
\end{tabular}

Results are mean (SEM) or numbers of patients.

${ }^{\star}$ Modified Canadian neurological score. formed using a previously described semiautomated and validated analysis. ${ }^{4}$ The number of transaxial slices over which the perfusion defect extended was defined initially. The slice with the largest perfusion defect was selected and an elliptical region of interest was placed over the hypoperfused region; as the analysis was automated, the same region of interest was subsequently used to analyse the remaining transaxial slices to avoid manual region of interest placement. On each transaxial slice the region of interest was mirrored from the hypoperfused hemisphere on to the contralateral hemisphere and the pixels in the affected hemisphere which had a count rate of $<12 \%$ of the mirror pixel on the normal side ${ }^{22}$ were identified. A lower cut off value (about $50 \%$ maximum count in the study) was set to eliminate asymmetries arising from the white matter and ventricles. A volumetric perfusion defect index was then calculated in which each voxel was weighted in proportion to the severity of hypoperfusion.

The formula (volume day 1 -volume day 2 /volume day 1) $\times 100$ was used to calculate percentage change in cerebral tissue perfusion. Regions of hyperperfusion were defined as (maximum) $100 \%$ reperfusion in view of the report of HMPAO hyperfixation relative to cerebral blood flow. ${ }^{23}$ If the perfusion defect increased in the second study, a negative reperfusion value was applied.

\section{STATISTICAL ANALYSIS}

As the Barthel score provided qualitative ranked data and was not normally distributed at three months, single and multiple linear regression analyses for non-parametric variables $^{24}\left(\chi^{2}\right)$ were used. The relation between the Barthel score at three months and the following covariates were analysed: age, sex, history of treated hypertension, heart disease, neurological score at admission, size of the perfusion defect on the SPECT study in the first 24 hours, and percentage reperfusion at 24 to 48 hours. Results were considered statistically significant at $\mathrm{P}<0.05$.

\section{Results}

ALL PATIENTS

During the study period 53 patients fulfilled the inclusion criteria (table 1). Twenty three additional patients were excluded because they had normal perfusion studies (21 patients) or had recurrent stroke during the follow up period (two patients). The mean time of the first ${ }^{99 \mathrm{~m}}$ Tc-HMPAO injection was 8.2 (SD 0.8 ) hours after the onset of symptoms, and the mean time of the second injection was 32.5 (SD 1.1) hours. Eight patients were treated with intravenous streptokinase during the pilot phase of the Australian streptokinase trial. ${ }^{1}$ Thirteen patients were participants in the blind phase of the trial (treated with either placebo or intravenous streptokinase) and seven patients were treated with intra-arterial streptokinase. ${ }^{16}$ At three months 13 patients had died and 14 patients had full functional recovery (Barthel score of 100). 


\section{UNIVARIATE ANALYSIS}

In univariate analysis, the following variables correlated significantly with the Barthel score at three months: age, neurological score at admission, perfusion defect size on the SPECT study in the first 24 hours, and percentage change in cerebral tissue perfusion at 24 to 48 hours (all $P<0.01$, table 2 ). The correlation of heart disease with functional outcome at three months approached significance $(P=0.07)$

\section{MULTIPLE LINEAR REGRESSION ANALYSIS}

In multiple linear regression analysis age $(P<0.01)$ and percentage change in cerebral tissue perfusion at 24 to 48 hours $(P<0.01$; table 3) provided independent prognostic information at three months. The admission neurological score $(P=0.07)$ approached significance as an independent outcome predictor at three months. The size of the perfusion defect on the SPECT study in the first 24 hours was not an independent outcome predictor, even after the analysis was performed with percentage change in cerebral perfusion excluded. The time of the first ${ }^{99 \mathrm{~m}} \mathrm{Tc}-\mathrm{HMPAO}$ injection was not a significant outcome predictor when it was included in the univariate and multivariate analyses.

\section{Discussion}

The finding that cerebral perfusion changes on 99m Tc-HMPAO SPECT in the first 24 to 48 hours are powerful independent predictors of outcome, strongly supports the notion that an early reperfusion window exists in humans. This has important implications for therapeutic trials for acute stroke. It is most likely that when an improvement in perfusion occurred on the second study, there had been embolic clearing or recruitment of the collateral circulation. However, it is acknowledged that many factors may affect ${ }^{99 \mathrm{~m}} \mathrm{Tc}$-HMPAO distribution including rheological factors, blood pressure, tracer redistribution depending on the time that scanning was performed after radio-

Table 2 Univariate analysis (non-parametric): correlation between variables and the Barthel score at three months

\begin{tabular}{lll}
\hline Variable & $\chi^{2}$ Statistic & $P$ value \\
\hline Age & 10.37 & $<0.01$ \\
Sex & 1.34 & 0.25 \\
Admission neurological score* & 12.95 & $<0.01$ \\
Hypertension & 0.01 & 0.93 \\
Heart disease & 3.39 & 0.07 \\
Perfusion defect size in first 24 hours & 7.94 & $<0.01$ \\
Percentage perfusion change at 24-48 hours & 17.44 & $<0.01$ \\
\hline
\end{tabular}

${ }^{\star}$ Modified Canadian neurological score.

Table 3 Multiple linear regression analysis (non-parametric): correlation between the variables and the Barthel score at three months

\begin{tabular}{lcc}
\hline Variable & $\chi^{2}$ Statistic & $P$ value \\
\hline Age & $7 \cdot 70$ & $<0.01$ \\
Sex & 0.19 & 0.66 \\
Admission neurological score* & 3.40 & 0.07 \\
Hypertension & 0.03 & 0.86 \\
Heart disease & 0.01 & 0.92 \\
Perfusion defect size in first 24 hours & 1.24 & 0.27 \\
Percentage perfusion change at 24-48 hours & 16.13 & $<0.01$ \\
\hline
\end{tabular}

pharmaceutical administration, drugs, or altered glutathione concentrations.

The exact time window during which purely nutritional reperfusion occurs has yet to be determined and is likely to be shorter than 24 to 48 hours and to be dependent on the combination of the duration and severity of ischaemia. ${ }^{25}$ In this study it was not possible to determine the exact time window during which purely nutritional reperfusion occurred; the time of the first ${ }^{99 m}$ Tc-HMPAO injection was not an independent predictor when it was included in the analysis. Based on experimental evidence in animals, severely ischaemic tissue, in which ionic disruption has occurred, may be viable for less than one to two hours. ${ }^{26}$ Mildly to moderately ischaemic tissue, in which electrical function is disturbed without loss of ionic homeostasis-"the ischaemic penumbra"27_may be viable for up to three hours in rat models of middle cerebral artery occlusion $^{28}$ and six to eight hours in baboons and squirrel monkeys. ${ }^{2529}$ Evidence from studies of stroke patients, using PET, suggests that in some patients ischaemic tissue may be viable for up to 48 hours or longer, based on the finding of the "misery perfusion" pattern of blood flow and metabolism..$^{30-31}$

Improved outcome and restriction of infarct size has been reported in patients in whom middle cerebral artery recanalisation, measured by transcranial doppler ultrasonography, occurred within eight hours in the presence of a good collateral circulation. ${ }^{32}$ In patients with middle cerebral artery reopening within the first 24 hours, reduced frequency of haemorrhagic transformation and oedema have been described. ${ }^{33}$ However, these studies did not use multiple linear regression analyses to account for other variables which may affect outcome and it is uncertain as to whether there is always a direct relation between arterial recanalisation and tissue reperfusion.

A study of shorter time windows using SPECT and other functional imaging techniques such as PET and echoplanar perfusion gadolinium enhanced imaging ${ }^{34}$ may allow a more precise quantification of the extent of the reperfusion time window. In such studies, measurements of the severity of blood flow reduction, together with measurements of tissue salvage, by coregistering structural and functional studies, may provide valuable additional information.

An important feature of this study was the use of multiple linear regression analyses to compare the prognostic value of SPECT measurements with established clinical measures. $^{8-13}$ A major difficulty with such analyses is that the outcome variable, the Barthel score at three months, is a qualitative ranked variable and is not normally distributed, as at three months many patients have died (score 0 ) or are functionally independent (score 100). For this study a non-parametric multiple linear regression analysis was used, ${ }^{23}$ which may be applied to future outcome studies.

The clinical variables of age, sex, admission neurological score, hypertension, and heart disease were included in the analysis as they 
have been shown to provide prognostic information $^{89}$ for acute and late outcome.

Perfusion defect size by SPECT was also included as some studies have shown a strong correlation with outcome in the past $^{35}$ although not universally. ${ }^{3637} \mathrm{~A}$ number of explanations may exist for this, including different methods for assessing defect size, different radiopharmaceuticals, and different times of study after stroke onset.

In this study, a correlation was found between the SPECT defect size in the first 24 hours in univariate analysis but this was not sustained in multiple linear regression analysis, even after reperfusion was removed as one of the covariates. This is probably because of a close relation between neurological score at admission and the size of the perfusion defect in the first 24 hours.

Because serial changes in cerebral perfusion within the first 24 to 48 hours are of independent prognostic value and because perfusion defect size in the first 24 hours is not, we hypothesise that haemodynamic changes occurring during this time may significantly affect outcome and possibly account for the disparate results between the studies described earlier.

In the setting of acute stroke therapies, functional imaging techniques may allow the reperfusion and therapeutic windows to be determined in humans. Given the strong predictive value of serial cerebral perfusion measurements within the first 48 hours after ischaemic stroke, therapeutic efforts aimed at further improving perfusion more during this time seem to be justified.

AEB is supported by the National Health and Medical Research Council of Australia. The project was supported by a grant from the Australian Stroke and Neuroscience Institute.

The staff of the Neurology and Nuclear Medicine Departments at the Austin Hospital are gratefully acknowledged. Ms K Diamantopoulou, Ms M Prince, and Dr P McCloud of the Department of Statistics, Monash University, Clayton, Victoria, Australia are thanked for their assistance with the statistical analyses.

This work was presented in part at the third international symposium on thrombolytic therapy in acute ischemic stroke, Nara, Japan, 1994.

1 Donnan GA, Davis SM, Chambers BR, et al. Australian streptokinase trial (ASK). In: del Zoppo GJ, Mori E, Hacke W, eds. Thrombolytic therapy in acute ischemic strok II. Berlin: Springer-Verlag, 1993:80-5.

2 Pulsinelli $W$. Pathophysiology of acute ischaemic stroke. Lancet 1992;339:533-6.

3 Marchal G, Serrati C, Rioux P, et al. PET imaging of cerebral perfusion and oxygen consumption in acute ischaemic stroke: relation to outcome. Lancet 1993;341 925-7.

4 Baird AE, Donnan GA, Austin MC, Fitt GJ, Davis SM McKay WJ. Reperfusion after thrombolytic therapy in ischemic stroke measured by single-photon emission computed tomography. Stroke 1994;25:79-85.

5 Herderscheê D, Limburg $M$, van Royen EA, Hijdra $A$, Büller HR, Koster PA. Thrombolysis with recombinant tissue plasminogen activator in acute ischemic stroke: tissue plasminogen activator in acute ischemic stroke:
evaluation with rCBF-SPECT. Acta Neurol Scand evaluation with

6 Overgaard K, Sperling B, Boysen G, et al. Thrombolytic therapy in acute ischemic stroke. A Danish pilot study. Stroke 1993;24:1439-46.

7 Hanson SK, Grotta JC, Rhoades H, et al. Value of singlephoton emission-computed tomography in acute strok therapeutic trials. Stroke 1993;24:1322-9.

8 Chambers BR, Norris JW, Bette LS, Hachinski VC. Prognosis of acute stroke. Neurology 1987;37:221-5.

9 Biller J, Love BB, Marsh III E, et al. Spontaneous improve- ment after acute ischemic stroke. A pilot study. Stroke 1990;21:1008-12.

10 Lefkovits J, Davis SM, Rossiter SC, et al. Acute stroke outcome: effects of stroke type and risk factors. Aust $N Z \mathcal{F}$ Med 1992;22:30-5.

11 Censori B, Camerlingo M, Casto L, et al. Prognostic factors in first-ever stroke in the carotid artery territory seen within 6 hours after onset. Stroke 1993;24:532-5.

12 Oxbury JM, Greenhall RCD, Grainger KMR. Predicting the outcome of stroke: acute stage after cerebral infarction. $B M \mathcal{F} 1975 ; 3: 125-7$.

13 Brass LM, Rattner Z. SPECT imaging in cerebrovascular disease. Brain SPECT perfusion imaging: image acquisition, processing, display and interpretation. In: Proceedings of a workshop held at Brookhaven National Laboratory 8-9 October 1991:77-88.

14 Heiss W-D, Emunds H-G, Herholz K. Cerebral glucose metabolism as a predictor of rehabilitation after ischemic stroke. Stroke 1993;24:1784-8.

15 Chambers BR, Donnan GA, Bladin PF. Patterns of stroke. An analysis of the first 700 consecutive admissions to the An analysis of the first 700 consecutive admissions to the Austin

16 Fitt GJ, Farrar J, Baird AE, et al. Pilot study of intra-arterial streptokinase in acute ischaemic stroke. Med $\mathcal{F}$ Aust 1993;159:331-4

17 Côté R, Battista RN, Wolfson C, Boucher J, Adam J, Hachinski V. The Canadian neurological scale: validation and reliability assessment. Neurology 1989;39: 638-43.

18 Mahoney FI, Barthel DW. Functional evaluation: the Barthel index. Md St Med f 1965;14:61-5.

19 Granger CV, Dewis LS, Peters NC, Sherwood CC, Barett JE. Stroke rehabilitation: analysis of repeated Barthel index measures. Arch Phys Med Rehabil 1979;60:14-7.

20 Marquardsen J. Natural history and prognosis of cerebrovascular disease. In: Ross Russell RW, ed. Cerebral arterial disease. Edinburgh: Churchill Livingstone, 1976: arterial

21 Chang LT. A method for attenuation correction in radionuclide computed tomography. IEEE Transactions on Nuclear Science 1978;NS-25:638-43.

22 Podreka I, Suess E, Goldenberg G, et al. Initial experience with technetium-99m HM-PAO brain SPECT. $\mathcal{f}$ Nucl Med 1987;28:1657-66.

23 Sperling B, Lassen NA. Hyperfixation of HMPAO during the subacute phase of stroke leading to spuriously high

24 Adichie JN. Rank tests in linear models. In: Krishnaiah PR, Sen PK, eds. Handbook of statistics 4: non-parametric methods. Amste

25 Jones TH, Morawetz RB, Crowell RM, et al. Thresholds of focal cerebral ischemia in awake monkeys. $\mathcal{f}$ Neurosurg 1981;54:773-82.

26 Siesjö BK. Pathophysiology and treatment of focal cerebral ischemia part I: pathophysiology. F Neurosurg 1992;77: 169-84.

27 Astrup J, Siesjš BK, Symon L. Thresholds in cerebral ischemia-the ischemic penumbra. Stroke 1981;12: 723-5.

28 Kaplan B, Brint S, Tanabe J, Jacewicz $M$, Wang X, Pulsinelli W. Temporal thresholds for neocortical infarction in rats subjected to reversible focal cerebral ischemia. Stroke 1991;22:1032-9.

29 Crowell RM, Marcoux FW, DeGirolami U. Variability and reversibility of focal cerebral ischemia in unanesthetized monkeys. Neurology 1981;31:1295-302.

30 Heiss W-D, Huber M, Fink GR, et al. Progressive derangement of periinfarct viable tissue in ischemic stroke. $f$ mereb Blood Flow Metab 1992;12:193-203.

31 Baron JC. Pathophysiology of acute cerebral ischemia: PET studies in humans. Cerebrovasc Dis 1991;1 (suppl 1): 22-31.

32 Ringelstein EB, Biniek R, Weiller C, Ammeling B, Nolte $P N$, Thron A. Type and extent of hemisphere brain infarctions and clinical outcome in early and delayed middle cerebral artery recanalization. Neurology 1992;42: 289-98.

33 von Kummer $R$, Forsting $M$. Effects of recanalization and collateral blood supply on infarct extent and brain edema after middle cerebral artery occlusion. Cerebrovasc Dis 1993;3:252-5.

34 Warach S, Chien D, Li W, Ronthal M, Edelman RR. Fast magnetic resonance diffusion-weighted imaging of acute human stroke. Neurology 1992;42:1717-23.

35 Limburg M, van Royen EA, Hijdra A, Verbeeten B Jr. rCBF-SPECT in brain infarction: when does it predict rCBF-SPECT in brain infarction: wher

36 Defer G, Moretti JL, Cesaro P, Sergent A, Raynaud C, Degos JD. Early and delayed SPECT using N-isopropyl p-iodoamphetamine iodine 123 in cerebral ischemia. A prognostic index for clinical recovery. Arch Neurol 1987; 44:715-8

37 Lee RGL, Hill TC, Holman BL, Royal HD, O'Leary DH, Clouse ME. Predictive value of perfusion defect size using $N$-isopropyl-(I-123)-p-iodoamphetamine emission tomography in acute stroke. $\mathcal{F}$ Neurosurg 1984;61: 449-52. 